Divulgación Científica

\title{
GRAFENO
}

\section{ALÓTROPO DEL CARBONO}

Hasta el siglo pasado se hablaba del grafito, diamante y fullerenos como formas alotrópicas del átomo de carbono. Sin embargo, en tiempo reciente, esta lista se ha incrementado con otros dos alótropos, grafeno y nanotubos.

En el grafeno, los átomos de carbono tienen hibridación $\mathrm{sp}^{2}$, esto explica el ángulo de enlace, $120^{\circ}$, y el que los átomos de carbono se dispongan en un plano; además, el traslape de los orbitales híbridos $\mathrm{sp}^{2}$ forma enlaces sigma $\mathrm{C}-\mathrm{C}$ dando lugar a su estructura hexagonal, los electrones $p$, "sobrantes" de cada átomo de carbono, se solapan lateralmente originando un gigante orbital molecular pi, deslocalizado. Esta estructura se podría considerar como aromática.

La disposición descrita, origina una capa de átomos de carbono, densamente empaquetada, que al utilizar el microscopio electrónico de barrido, la imagen obtenida recuerda a la molécula de benceno. Es una estructura bidimensional de un átomo de grosor, formada por celdas hexagonales.

El premio Nobel de física de 2010, fue otorgado a Andre Geim y a Konstantin Novoselov, "por sus trabajos pioneros en el desarrollo del grafeno, un material bidimensional cuyas láminas solo tienen el espesor de u átomo de carbono", según lo afirmado por la Real Academia de Ciencias de Suecia.
Se afirma que este material es más fuerte que el acero, con propiedades electro - ópticas extraordinarias y puede revolucionar la ingeniería, informática y la electrónica. Sin embargo, según investigadores de Rice University de Houston y Tsinghua University de Beijín, aseguran que hay láminas $u$ hojas que no son lo fuertes que se dice, esto parece que se debe a que en los límites de la hoja se presentan anillos de siete o cinco átomos de carbono interrumpiendo el tramado hexagonal.

Por sus características estructurales, el grafeno tiene una amplia gama de propiedades y por ende de aplicaciones.

*. El hecho de que solo posea un elemento, evita la aparición de defectos estructurales como grietas que en otros materiales, son la causa de su rotura. Aspecto único en un material de estas características.

*. El grafeno es metálico, pudiéndose inducir electrones, teniendo movilidades electrónicas extraordinariamente altas en comparación con los conductores convencionales. Además independientemente de la temperatura, la movilidad electrónica se mantiene. Se ha alcanzado una movilidad de electrones de 10 elevado a 8 electrones por $\mathrm{cm}^{2}$.

* Su conductividad térmica es la más alta que la de cualquier otro material.

*. Los electrones pueden circular libremente sin riesgo de colisión con impurezas 
*. Puede ser estirado de forma reversible hasta un $20 \%$ sin sufrir ningún tipo de deformación.

*. Excelente conductor de la electricidad sin interferencias de clase alguna

*. Impermeable

*: Transparente y muy liviano

*. Es extremadamente flexible

*. Ecológico

Sus aplicaciones van desde cambios en la industria de te telefonía móvil, las telecomunicaciones pasando por la fabricación de chips hasta la forma de elaboración de fármacos contra el cáncer.

Una aplicación, que parece trascendental, por sus efectos, es la fabricación de membranas de grafeno.

En la universidad de Colorado se muestran grandes avances en la creación de membranas eficaces energéticamente para producir gas natural y, en forma concomitante, reducir emisiones de dióxido de carbono en las chimeneas térmicas o tubos de escape de automotores

El equipo de científicos está conformado por Scott Bunch, John Pellegrino, Steven Koenig y Luda Wang, de la Universidad de Colorado en Boulder, Estados Unidos, en el desarrollo de la investigación crearon poros manométricos en láminas de grafeno mediante un proceso de grabado oxidativo inducido por luz ultravioleta, luego se midió la permeabilidad a varios gases en las membranas de grafeno poroso.

El equipo de científicos realizó experimentos con diversos gases, tales como, hidrógeno, dióxido de carbono, argón, nitrógeno, metano y hexafluoruro de azufre, estas moléculas están en el intervalo entre 0,29 y 0,49 nanómetros, demostrando así el potencial que tiene el nuevo descubrimiento para permitir se- parar gases basándose en el tamaño molecular.

En el mismo sentido, los científicos afirman que el grafeno es un material ideal para crear una membrana de separación debido a sus características especiales, ya que incluyen su durabilidad y que no necesitan mucha energía para "empujar" a las moléculas por la membrana. La investigación sugiere que filtros de grafeno podrían superar a otras técnicas de desalación en un margen significativo.

Aún habrá que superar otros desafíos técnicos antes de que la tecnología pueda utilizarse en la práctica. Uno de ellos, es desarrollar un proceso práctico para crear con la debida precisión nanoporos de los tamaños deseados.

Varias potenciales aplicaciones del grafeno están en desarrollo y muchas más han sido propuestas. Estas incluyen la obtención de materiales livianos, flexibles, pantallas de visualización, finas y duraderas, circuitos eléctricos, células solares, así como diversos procesos industriales mejorados por el uso de nuevos materiales de grafeno médicos y químicos.

El grafeno tiene propiedades ideales para ser un excelente componente de los circuitos integrados, tiene una alta movilidad de portador, así como de bajo nivel de ruido, lo que le permite ser utilizado como el canal en un transistor de efecto de campo. La cuestión es que una sola hoja de grafeno es difícil de producir, y aún más difícil de hacer en la parte superior de un sustrato apropiado. En junio de 2011, los investigadores de IBM anunciaron que habían logrado crear el primer circuito integrado de grafeno basado en un mezclador de radio de banda ancha. El circuito maneja frecuencias de hasta $10 \mathrm{GHz}$, y su rendimiento no se afectó por temperaturas de hasta $127^{\circ} \mathrm{C}$. 
La gran conductividad eléctrica del grafeno y su alta transparencia óptica, hacen que sea un candidato para la elaboración de electrodos conductores transparentes, requeridos para aplicaciones tales como pantallas táctiles, pantallas de cristal líquido, células fotovoltaicas orgánicas, y los diodos emisores de luz orgánicos. En particular, la fuerza y la flexibilidad mecánica de grafeno son ventajosas en comparación con óxido de indio y estaño.

En 2011, los investigadores de Instituto de Tecnología de Georgia informaron que múltiples capas de grafeno alineadas verticalmente, en tres dimensiones, pueden ser clave para los materiales interfaciales térmicos basados en el grafeno, con superior conductividad térmica equivalente y muy baja resistencia térmica interfacial entre el grafeno y el metal.

Debido a la extremadamente alta relación superficie/masa del grafeno, una aplicación potencial es en las placas conductoras de ultracondensadores. Se cree que el grafeno podría ser utilizado para producir ultracondensadores con una mayor densidad de almacenamiento de energía que la que está disponible actualmente.

La teoría de simulaciones de densidad predice que depositar ciertos átomos sobre el grafeno, puede hacer un piezoeléctrico sensible a un campo eléctrico aplicado en la dirección vertical (es decir, perpendicular al plano). Este tipo de diseño es similar, en magnitud, al de los materiales piezoeléctricos a granel, hacen del grafeno una herramienta para el control y la detección de dispositivos a nanoescala.

Como se puede apreciar por lo anterior, las posibilidades de utilización de este alótropo de carbono son extremadamente variadas $y$, muy seguramente, van a trasformar, en forma positiva, la vida, desarrollo y bienestar de la sociedad.

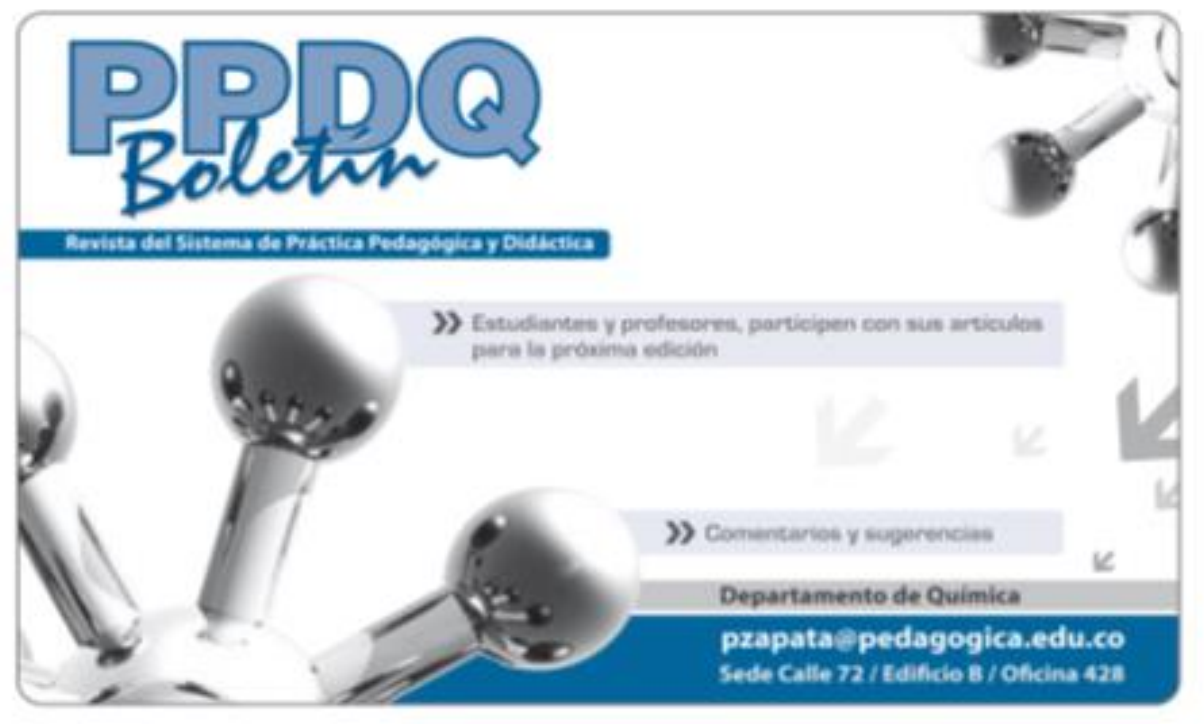

